\title{
Substâncias da natureza com atividade anti-Trypanosoma cruzi
}

\author{
Dênia A. Saúde-Guimarães*, Angélica R. Faria \\ Laboratório de Estudos Químicos e Farmacológicos de Plantas Medicinais, Departamento de Farmácia, Escola \\ de Farmácia, Universidade Federal de Ouro Preto, Rua Costa Sena 171, Centro, 35400-000, Ouro Preto, \\ Minas Gerais, MG, Brasil
}

\begin{abstract}
RESUMO: A doença de Chagas, uma zoonose causada por Trypanosoma cruzi, afeta em todo o mundo cerca de 16 a 18 milhões de pessoas. Sua transmissão ocorre através das fezes de triatomíneos, insetos hematófagos conhecidos como barbeiro. Atualmente, a principal forma de transmissão da doença de Chagas em áreas urbanas é por meio de transfusão de sangue contaminado. A violeta de genciana é o único agente que pode ser empregado na quimioprofilaxia de sangue destinado à transfusão. No entanto, existem algumas restrições ao seu uso. A quimioterapia disponível para a doença de Chagas não é eficaz uma vez que as drogas disponíveis nifurtimox e benznidazol são ativas apenas na fase aguda da doença e apresentam sérios efeitos colaterais. Várias substâncias isoladas de plantas foram avaliadas como agentes anti-T. cruzi, objetivando encontrar drogas com menos efeitos colaterais e maior eficácia para a quimioprofilaxia e quimioterapia da doença de Chagas. Nesta revisão são apresentadas as substâncias de origem natural com atividade anti- $T$. cruzi.
\end{abstract}

Unitermos: Trypanosoma cruzi, doença de Chagas, substâncias naturais.

\begin{abstract}
S: “Natural compounds with anti-Trypanossoma cruzi activity". Chagas'disease, a zoonose caused by Trypanosoma cruzi, affects 16-18 million people in the world. The most important mode of transmission of the disease is associated with the feces of several species of triatomine bugs that are strictly hematophagous. Actually, infected blood transfusion is the major mechanism of transmission in urban areas. Gentian violet is the only available prophylactic drug. Despite its effectiveness, there are some restrictions on its use. The available therapy of Chagas' disease is inadequate since the treatment of patients with the drugs nifurtimox and benznidazole presents serious toxic side effects. The search for new trypanocidal compounds to the treatment of Chagas'disease and to use for eliminating T. cruzi from blood, that are more effective and that do not affect red blood cells is the main goal in the prevention of Chagas' disease. In this review a large set of chemicals of plant origin are enumerated and their trypanocidal activity are briefly discussed.
\end{abstract}

Keywords: Trypanosoma cruzi, Chagas’ disease, natural compounds.

\section{INTRODUÇÃO}

A doença de Chagas, uma zoonose causada por Trypanosoma cruzi, afeta em todo o mundo cerca de 16 a 18 milhões de pessoas, das quais 50 mil morrem a cada ano (Sigman, 2006). Apenas nas América Central e do Sul, existem em torno de 8 a 9 milhões de pessoas infectadas e 25 milhões sob o risco de infecção (WHO, 2002). A transmissão da doença de Chagas ocorre através das fezes de triatomíneos, insetos hematófagos conhecidos como barbeiro. No Brasil está forma de transmissão é controlada pelo uso de inseticidas. Atualmente, a principal forma de transmissão da doença de Chagas em áreas urbanas é através da transfusão de sangue contaminado, cujo risco é de 20\% (Schmunis, 1999). Esse tipo de transmissão adquiriu especial relevância em regiões que não possuem o vetor, mas que recebem imigrantes de áreas afetadas. Outra forma de transmissão da doença ocorre de maneira esporádica e circunstancial, através de alimentos contaminados com o parasita, principalmente a partir de triatomíneos ou de suas dejeções. Também, pode ocorrer através da ingestão de carne crua ou mal cozida de caça, ou de alimentos contaminados por urina ou secreção anal de marsupiais infectados, ou mesmo por meio de hábitos primitivos de ingestão de triatomíneos (Dias, 2006).

A necessidade de se prevenir à transmissão da doença por transfusão levou à busca por drogas que eliminem o parasita no sangue, ou seja, uma abordagem quimioprofilática. Esta consiste na esterilização do sangue por substâncias que permitam transfundi-lo, com segurança, entre seres humanos. A violeta de genciana é o único agente empregado na quimioprofilaxia de sangue destinado a esse fim. No entanto, existem algumas restrições ao seu uso, pois este corante triarilmetânico confere ao sangue uma coloração púrpura, podendo manchar pele e mucosas, causar a microaglutinação e a aglomeração dos eritrócitos, além 
de apresentar propriedades mutagênicas (Dias; Wendel, 1992). O uso da violeta de genciana requer um tempo de exposição para a esterilização do sangue de 24 horas, tempo considerado longo para pequenos postos onde há carência de sangue estocado, bem como para aquelas situações de emergência, quando a transfusão de sangue requer ação imediata (Chiari et al., 1996). Assim, há necessidade da busca por novas drogas mais eficazes e com menores efeitos colaterais que a violeta de genciana e por novos procedimentos para a quimioprofilaxia da doença de Chagas adquirida por transfusão.

Ao longo dos anos, uma grande variedade de drogas foi investigada com o objetivo de estabelecer uma quimioterapia mais efetiva para a doença de Chagas. Os ensaios terapêuticos com nifurtimox foram iniciados em 1965 e com benznidazol em 1971. Estas drogas eliminam os parasitas na fase aguda da doença, diminuindo o curso da infecção. Assim, em alguns casos, pacientes são curados com o tratamento, fato que se deve, em parte, à heterogeneidade da população de parasitas. Um estudo realizado com cem portadores da forma indeterminada da doença, tratados com nifurtimox e benznidazol no período de 1965 a 1985, revelou que a freqüência de cura foi de apenas 6\% para o primeiro e de $10 \%$ para o segundo (Ferreira, 1990). Ambas as drogas causam sérios efeitos colaterais, tais como anorexia, náusea, vômito, dor de cabeça, depressão do sistema nervoso central ou sintomas maníacos, vertigem, parestesias, polineuropatias periféricas e dermatites. Fato este que é particularmente problemático, visto que um grande número de doses deve ser administrado por um período longo para que o tratamento obtenha sucesso. Durante algum tempo estas drogas foram usadas no tratamento de pacientes chagásicos em fase aguda da doença, mas atualmente, o benznidazol é a única droga utilizada (Castro,1993). Vale ressaltar que esse fármaco é eficaz se utilizado na fase aguda da doença e em quadros clínicos que não acometam o sistema cardiovascular. Várias cepas de T. cruzi foram isoladas de diferentes países e zonas geográficas. Diferenças importantes na resistência ou suscetibilidade às drogas em uso, em experimentação em laboratório, ou em estudos clínicos, foram relatados dentre as diferentes cepas do parasita. Esta situação dificulta a busca por novas drogas antiT. cruzi (Morelo et. al., 1994). Assim, a carência de medicamentos mais eficazes para a cura da infecção nas suas fases aguda e crônica permanece (Figura 1).

\section{Substâncias naturais com atividade anti-T. Cruzi}

A tripanotiona, um metabólito encontrado unicamente no parasita tripanossomatídeo, e a sua enzima tripanotiona redutase desempenham além de outras importantes funções, papel central na manutenção do balanço tiol-redox da célula e, conseqüentemente, atraíram muito interesse como alvo quimioterapêutico contra a doença de Chagas (Fairlamb, 1994). O pré-tratamento, de formas tripomastigotas, com naftoquinonas e nitrofuranos levaram à redução na percentagem de células infectadas em culturas de músculo liso. Na presença de oxigênio, estes compostos não inativaram a tripanotiona redutase, mas produziram alterações em sua função protetora na regeneração de radicais livres. Como a tripanotiona é um intermediário crítico no metabolismo de tripanossomatídeos e está ausente no hospedeiro, inibidores desta enzima podem constituir agentes anti-T. cruzi muito promissores (Castro, 1993).

Substâncias de origem natural de diversas classes, como quinonas, flavonóides e terpenos mostraram-se ativas contra o T. cruzi, e são apresentadas a seguir.

\section{Quinonas}

A naftoquinona $\beta$-lapachona, presente em várias espécies vegetais da família Bignoniaceae, apresentou atividade contra as formas amastigota, epimastigota e tripomastigota do parasita. O seu derivado de transformação química, a 3-alil- $\beta$-lapachona, foi ativo contra formas tripomastigotas e tem sido sugerido como uma droga alternativa para o uso em bancos de sangue (Castro, 1993). Já a plumbagina, isolada de Pera benensis Rusby (Euphorbiaceae), foi $100 \%$ ativa na concentração $250 \mu \mathrm{g} / \mathrm{mL}$ de sangue contaminado e sua atividade foi comparada com a da violeta de genciana (Fournet et al., 1994) (Figura 2).

A espécie Oxalis erythrorhiza Gillies ex Hooker et Arnott, conhecida na província de San Juan, Argentina, como "boldo da cordilheira”, é utilizada na medicina popular para problemas cardíacos e hepáticos. Das suas partes aéreas foram isoladas, a benzoquinona embelina e também alguns alquil fenóis. A embelina mostrou atividade contra o T. cruzi, com $100 \%$ de lise dos protozoários, em uma concentração de $100 \mu \mathrm{g} / \mathrm{mL}$ (Feresin et al., 2003) (Figura 3).

\section{Flavonóides}

O 3-metoxiflavonol penduletina e a flavanona sacuranetina, isolados de Trixis vauthieri L. (Asteraceae), eliminaram 99 e 100\%, respectivamente, do parasita no sangue infectado, na concentração de 500 $\mu \mathrm{g} / \mathrm{mL}$. O cristal de violeta, utilizado como substância de referência, eliminou 50\% do parasita na dosagem de $31 \mu \mathrm{g} / \mathrm{mL}$ no mesmo ensaio (Ribeiro et al., 1997).

Grael et al. (2005) isolaram sete substâncias ativas contra formas tripomastigotas de $T$. cruzi a partir de extratos de Lychnophora pohlii Schultz-Bip (Asteraceae). Do extrato metanólico, foram isolados os flavonóides luteolina e vicenina-2. Esses flavonóides demonstraram atividade tripanossomicida significativa na concentração de $500 \mu \mathrm{g} / \mathrm{mL}$. Por comparação, essas substâncias foram menos ativas que a violeta de genciana 
( IC $_{50} 76 \mu \mathrm{mol} / \mathrm{L}$ e $31 \mu \mathrm{g} / \mathrm{mL}$ respectivamente).

O extrato de Lychnophora staavioides Mart.

(Asteraceae) forneceu os flavonóides: tectochrysina (1), pinostrobina (2), pinobanksina (3), acetato de pinobanksina (4), pinocembrina (5), chrysina (6), galangina-3-metil éter (7), quercetina-3-metil éter (8), chrysoeriol (9) e vicenina-2 (10) que foram testados contra o T. cruzi. A substância mais ativa foi a quercetina-3-metil éter, que não causou lise de células sanguíneas e mostrou-se promissora para uso contra $T$. cruzi em bancos de sangue. Esta substância apresentou uma porcentagem de atividade tripanossomicida de $63,2 \pm 4,1 \%$, já a tectochrysina demonstrou 38,5 \pm 8,3 \% e o chrysoeriol 32,7 $\pm 7,1 \%$ de redução dos parasitas sangüíneos. Todas estas substâncias foram ativas na dose de $500 \mu \mathrm{g} / \mathrm{mL}$ (Takeara et al., 2003).

Do extrato clorofórmico de Moquinia kingii foi obtida a apigenina que apresentou ação contra tripomastigotas in vitro (Schinor et al., 2004). O valor da IC $_{50}$ desta substância foi $181,6 \mu \mathrm{g} / \mathrm{mL}$ ou $0,68 \mathrm{mM}$ e a IC $_{50}$ para violeta genciana, usado como controle positivo, foi de $31 \mu \mathrm{g} / \mathrm{mL}$ ou $76 \mu \mathrm{M}$ (Figura 4).

\section{Alcalóides}

Fournet e colaboradores realizaram um estudo no qual nove alcalóides bisbenzilisoquinolínicos foram testados. Sete dos nove alcalóides testados foram $100 \%$ ativos. São eles: cocsolina, dafnandrina e dafnolina, isolados de Albertisia cf. A. papuana Becc (Menispermaceae), limacina, obtida de Caryomene olivascens, girocarpina e feantina, isolados de Gyrocarpus americanus e isochondrodendrina, oriundo de Curarea candicans (L.C. Rich) Barneby and Krukoff (Menispermaceae) (Fournet et al., 1994) (Figura 5).

Estudos avaliaram os efeitos anti-T. cruzi do alcalóide piperina, principal metabólito secundário em Piper nigrum, conhecida popularmente como pimenta preta. A piperina foi testada contra epimastigotas e amastigotas do T. cruzi, demonstrando toxicidade dosedependente, com $\mathrm{IC}_{50}$ de 7,36 $\mu \mathrm{M}$ para epimastigotas e $\mathrm{IC}_{50}$ de 4,91 $\mu \mathrm{M}$ para amastigotas. Dessa maneira, a piperina demonstrou ser uma potente substância tripanossomicida, sendo mais tóxica para amastigotas intracelulares que para epimastigotas (Ribeiro et al., 2004) (Figura 6).

Os alcalóides 2-n-propilquinolina, chimanina B e chimanina D, isolados de Galipea longiflora Krause (Rutaceae), apresentaram atividades similares às das drogas de referência nifurtimox e benznidazol, contra cinco cepas de formas epimastigotas do T. cruzi (Fairlamb, 1994). A 2-n-propilquinolina e a chimanina $\mathrm{D}$ apresentaram $\mathrm{IC}_{90}$ de $50 \mu \mathrm{g} / \mathrm{mL}$ para as cinco cepas de formas epimastigotas. Chimanina $B$ mostrou $\mathrm{IC}_{90}$ de $25 \mu \mathrm{g} / \mathrm{mL}$ para as cinco cepas. As drogas de referência nifurtimox e benznidazol apresentaram $\mathrm{IC}_{90}$ variando de 25 a 100 e de 25 a $50 \mu \mathrm{g} / \mathrm{mL}$, respectivamente
(Figura 7).

\section{Terpenos}

O ácido caurenóico isolado de Mikania obtusata DC, Asteraceae (Alves et al., 1995), Xylopia frutescens Aubl., Annonaceae (Takahashi et al., 1994) e de Viguiera aspillioides Gardn., Asteraceae (Costa et al., 1995), apresentou $100 \%$ de atividade contra o T. cruzi, na concentração de $1000 \mu \mathrm{g} / \mathrm{mL}$ de sangue contaminado.

O ácido xilópico, diterpeno isolado de Xylopia frutescens Aubl., Annonaceae (Takahashi et al., 1994) e o caurenol, isolado de Viguiera aspillioides Gardn., Asteraceae (Costa et al., 1995) e de Xylopia frutescens Aubl., Annonaceae (Takahashi et al., 1994), foram ativos nas concentrações de 500 e $1000 \mu \mathrm{g} / \mathrm{mL}$.

Os diterpenos 17-hidroxicauranol e o ácido traquilobânico, isolados de Viguiera aspillioides Gardn. (Asteraceae) foram ativos nas concentrações de 500 e $1000 \mu \mathrm{g} / \mathrm{mL}$ de sangue contaminado (Costa et al., 1995) (Figura 8).

Nascimento et al. (2004) avaliaram as atividades tripanossomicidas in vitro de terpenóides isolados de Mikania stipulacea e Mikania hoehnei, espécies vegetais conhecidas popularmente como guaco. O diterpeno ácido ent-9 $\alpha$-hidroxi-15 $\beta$-E-cinnamoiloxi-16-cauren19-óico apresentou atividade contra tripomastigotas, reduzindo o número de parasitas em 61,7, 62,8 e 69,4\% nas concentrações de 100, 250 e $500 \mu \mathrm{g} / \mathrm{mL}$, respectivamente. O gênero Mikania é conhecido por conter diterpenos caurenos, particularmente o ácido caurenóico, que demonstrou atividade parcial contra $T$. cruzi.

A espécie Dracocephalum komarovi Lipsky (Labiatae) é bastante usada no Uzbequistão na forma de chá para tratamento de doenças inflamatórias. Desta espécie foram isolados os diterpenos komarovispirona, dracocequinonas A e $B$ que foram ativos contra epimastigotas com concentração letal mínima de 23, 12,5 e $25 \mu \mathrm{M}$, respectivamente (Uchiyama et al., 2004) (Figura 8).

Das partes aéreas de Alomia myriadenia, foram isolados os diterpenos ent-8S,12S-epoxi-7R,16diidroxihalima-5(10),13-dien-15,16-olídeo, ent16-hidroxilabda-7,13-dien-15,16-olídeo e ent-12Rhidroxilabda-7,13-dien-15,16-olídeo. Da avaliação destes três diterpenos, o último foi o que se mostrou mais ativo, quando testado in vitro contra formas tripomastigotas de $T$. cruzi em sangue infectado de murinos, causando a lise de $100 \%$ dos parasitas na concentração de $250 \mu \mathrm{g} / \mathrm{mL}$ (Scio et al., 2003) (Figura 8).

O taxol, um diterpeno com atividade antitumoral, isolado de Taxus brevifolia (Taxaceae), foi ativo, interferindo na proliferação dos epimastigotas, impedindo que a divisão celular se completasse, mas 
permitindo a multiplicação de organelas. O triterpeno tingenona, também extraído da mesma espécie, foi ativo contra epimastigotas, causando inibição da síntese de proteínas e ácidos nuclêicos no T. cruzi (Castro, 1993). Outros terpenos foram avaliados por Takahashi et al., 2002 e Leite et al., 2001) (Figura 8).

Estudos realizados com 55 espécies vegetais da família Asteraceae, visando obter substâncias com atividade tripanossomicida levaram a onze espécies ativas. Dessas, L. villosissima Mart., Lychnophora passerina (Mart ex. DC) Gardn, Lychnophora pinaster Mart., Lychnophora trichocarpha Spreng. e Hololepis pedunculata Backer já tiveram seus estudos fitoquímicos realizados (Tavares, 1990; Oliveira et al., 1996; Chiari et al., 1991 e 1996). Dentre outros constituintes químicos isolados destas quatro espécies, destacam-se as lactonas sesquiterpênicas 15-desoxigoiazensolídeo, goiazensolídeo, licnofolídeo, eremantolídeo C, isocentraterina e licnoforolídeo A. O goiasensolídeo, obtido de L. passerina (Mart ex. DC) Gardn, foi 100 \% ativo na concentração de $240 \mu \mathrm{g} / \mathrm{mL}$. O licnofolídeo, que inibiu $50 \%$ do crescimento dos tripomastigotas na concentração de $150 \mu \mathrm{g} / \mathrm{mL}$ e o eremantolídeo C, $100 \%$ ativo na concentração de $3600 \mu \mathrm{g} / \mathrm{mL}$, foram isolados de L. trichocarpha Spreng. (Oliveira et al., 1996; Saúde et al., 1998). Licnoforolídeo A e isocentraterina, isolados de Hololepis pedunculata Backer (Almeida, 1997), inibiram $100 \%$ do crescimento dos tripomastigotas nas concentrações de 0,38 e 0,51 mg/mL, respectivamente. De $L$. pinaster Mart isolou-se o ácido licnofóico, que inibiu $50 \%$ do crescimento dos tripomastigotas na concentração de $12 \mu \mathrm{g} / \mathrm{mL}$ (Oliveira et al., 1996).

Cinaropicrina, lactona sesquiterpência isolada do extrato clorofórmico de Moquinia kingi, foi testada contra formas tripomastigotas (Schinor et al., 2004). Tanto o extrato como a cinaropicrina mostraram ação tripanossomicida, sendo a $\mathrm{IC}_{50} 93,5 \mu \mathrm{g} / \mathrm{mL}$ ou $0,26 \mathrm{mM}$ para cinaropicrina e $\mathrm{IC}_{50} 31 \mu \mathrm{g} / \mathrm{mL}$ ou $76 \mu \mathrm{M}$ para violeta de genciana, usada como controle positivo,.

A desidrozaluzanina C, lactona sesquiterpênica isolada de Munnozia maronii (Asteraceae), apresentou $\mathrm{IC}_{90}$ em quinze diferentes cepas de epimastigotas variando entre 5 e $50 \mu \mathrm{g} / \mathrm{mL}$. Nifurtimox e benznidazol mostraram $\mathrm{IC}_{90}$ maior que $100 \mu \mathrm{g} / \mathrm{mL}$. Desidrozaluzanina C foi, portanto, mais ativa que as drogas de referência em todas as cepas (Fournet et al., 1993) (Figura 8).

Os sesquiterpenos hidroperóxidos (11 a 13), obtidos de Pogostemon cablin (Blanco) Benth., espécie originada da Ásia, mostraram atividade contra epimastigotas do T. cruzi com concentração letal mínima de $0,84 \mu \mathrm{M}$ para 11 e de 1,7 $\mu \mathrm{M}$ para 12 e 13 (Kuichi et al., 2004) (Figura 8).

\section{Xantonas}

Garciniaxantona B, isolada de Garcinia subelliptica e 8-Desoxigartanina, obtida das folhas de $G$. intermédia foram ativas contra formas epimastigotas nas concentrações de $66 \mu \mathrm{M}$ e $118 \mu \mathrm{M}$ e contra tipomastigotas nas concentrações de $8 \mu \mathrm{M}$ e $131 \mu \mathrm{M}$, respectivamente (Abe et al., 2004) (Figura 9).

\section{Neolignanas}

As neolignanas tetrahidrofurânicas grandsina, $\left(7 R, \quad 8 R, \quad 7^{\prime} R, \quad 8^{\prime} R\right)-3$ ',4'-metilenodioxi-3,4,5,5'tetrametoxi-7,7'-epoxilignana (14) e $\left(7 R, 8 R, 7^{\prime} R\right.$, 8'R)-3,4,3',4'-dimetilenodioxi-5,5'-dimetoxi-7,7' epoxilignana (15), isoladas das inflorescências de Piper solmsianum, apresentaram atividade contra epimastigotas do $T$. cruzi com IC $_{50}$ igual a 8,74, 17,6 e 3,47 $\mu \mathrm{g} / \mathrm{mL}$, respectivamente (Martins et al., 2003) (Figura 10).

\section{Benzofuranodiona}

O ácido úsnico, isolado do líquen Cladonia substellata, foi ativo contra epimastigotas do T. cruzi em concentrações de 5 a $30 \mu \mathrm{g} / \mathrm{mL}$ (De Carvalho et al., 2005). Análise dos epimastigotas tratados com esta substância mostrou danos a mitocôndrias e um aumento significativo no volume do cinetoplasto. Foi observada também a lise dos tripomastigotas em todas as concentrações testadas. Tratamento de macrófagos com 40 a $80 \mu \mathrm{g} / \mathrm{mL}$ de ácido úsnico levou a alterações significativas dos amastigotas intracelulares sem causar danos ultraestruturais significativos para a célula hospedeira (Figura 11).

\section{Peptídeos}

Seis peptídeos antimicrobianos, isolados da secreção cutânea das espécies de sapo Phyllomedusa hypochondrialis e P.oreades foram avaliados, in vitro, contra o T. cruzi (Leite et al., 2005). Esses peptídeos foram nomeados como phylloseptinas (PSs). Os ensaios utilizaram sangue fresco puncionado de ratos albinos suíços durante o pico de parasitemia (Bastos et al., 1999). Os testes revelaram que dois peptídeos, denominados PS4 e PS5 foram ativos contra T. cruzi em níveis micromolares. Os valores de $\mathrm{IC}_{50}$ para PS4, PS5 e violeta genciana foram 5,1, 4,9 e $76 \mu \mathrm{M}$, respectivamente. Devido ao fato desses peptídeos agirem diretamente eliminando os tripomastigotas, eles evitam que haja invasão das células hospedeiras pelo T. cruzi. Dessa maneira, essas substâncias seriam bastante úteis na prevenção da transmissão do T. cruzi em transfusões sanguíneas.

\section{CONCLUSÃO}

Pelas informações aqui contidas, torna-se evidente a necessidade de um programa interdisciplinar de pesquisa para o desenvolvimento de novas drogas 
eficazes na quimioprofilaxia e, principalmente, na a quimioterapia da doença de Chagas. Substâncias que ao serem adicionadas ao sangue eliminem os tripomastigotas, e possam substituir a violeta de genciana, que apesar de ser eficaz apresenta efeitos colaterais que dificultam seu uso. Além de drogas ativas na fase crônica, forma predominante da doença, capazes de atuar em diferentes cepas do parasita e que possam substituir nifurtimox e benznidazol, substâncias eficazes apenas na fase aguda, que apresentam sérios efeitos colaterais, além de serem genotóxicas e produzirem danos aos tecidos dos mamíferos. Nesse sentido, as plantas têm se mostrado uma rica fonte de substâncias com atividade contra as formas amastigota, epimastigota e tripomastigota do T. cruzi, e se apresentam como uma das direções promissoras na busca de drogas eficazes na prevenção e tratamento da doença de Chagas.

\section{REFERÊNCIAS}

Abe F, Nagafuji S, Okabe H, Akahane H, Estrada-Muñiz E., Huerta-Reyes M, Reyes-Chilpa R 2004. Trypanocidal constituents in plants 3 . Leaves of Garcinia intermedia and heartwood of Calophyllum brasiliense. Biol Pharm Bull 27: 141-143.

Almeida VL 1997. Estudo Químico e Atividade Tripanossomicida de Hololepis pedunculata Backer. Belo Horizonte, UFMG. Dissertação de Mestrado em Química.

Alves TMA, Chaves PPG, Santos LMST, Nagem TJ, Murta SMF, Ceravolo IP, Romanha AJ, Zani CL 1995. A diterpene from Mikania obtusata active on Trypanosoma cruzi. Planta Med 61: 85-87.

Bastos JK, Albuquerque S, Silva ML 1999. Evaluation of the trypanocidal activity of lignans isolated from the leaves of Zanthoxylum naranjillo. Planta Med 65: 541-544.

Castro SL 1993. The challenge of Chagas'disease chemotherapy: An update od drugs assayed against Trypanosoma cruzi. Acta Tropica 53: 83-98.

Chiari E, Oliveira AB, Raslan DS, Mesquita AAL, Tavares KG 1991. Screening in vitro of natural products against blood forms of Trypanossoma cruzi. T Roy Soc Trop Med $H$ 85: 372-374.

Chiari E, Duarte DS, Perry KSP, Saúde DA, Raslan DS, Boaventura MAD, Grandi TSM, Stehman JR, Oliveira AB 1996. In vitro screening of Asteraceae plant species against Trypanosoma cruzi. Phytother Res 10: 636-638.

Costa FB, Vichnewisk W, Albuquerque S 1995. Terpenóides bioativos de Viguiera aspillioides Gardn. (Asteraceae) com atividade tripanossomicida. 18 Reunião da Sociedade Brasileira de Química, 30 de maio a 2 de junho de 1995, Caxambu-MG.

De Carvalho EAB, Andrade PP, Silva NH, Pereira EC, Figueiredo RCBQ 2005. Effect of usnic acid from the lichen cladonia substellata on trypanosoma cruzi in vitro: an Ultrastructural Study. Micron 36: 155-<smiles>CN(C)c1ccc(C(=C2C=CCC=C2)c2ccc(N(C)C)cc2)cc1</smiles>

Violeta de Genciana<smiles>O=C(Cn1ccnc1[N+](=O)[O-])OCc1ccccc1</smiles><smiles>CC1C[Ge](=O)CCN1N=Cc1ccc([N+](=O)[O-])o1</smiles>

Figura 1. Medicamentos clássicos com atividade anti-Trypanosoma cruzi.<smiles>CC1([14CH3])CCC2=C(O1)c1ccccc1C(=O)C2=O</smiles><smiles>C=CCC1CC2=C(OC(CCCC)(CCCC)C2(C)O)c2ccccc2C1=O</smiles><smiles>CC1=CC(=O)c2c(O)cccc2C1=O</smiles>

Phumb agina

Figura 2. Quinonas com atividade anti-Trypanosoma cruzi. 


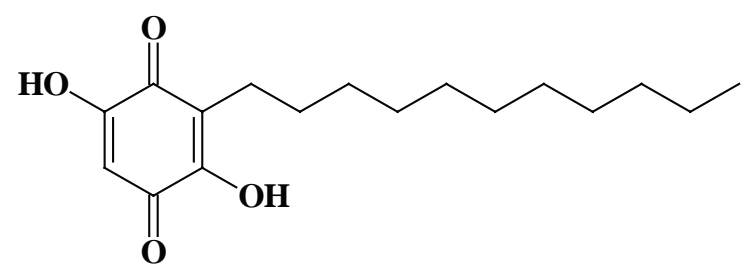

Embelina

Figura 3. Embelina de Oxalis erythrorhiza com atividade anti-Trypanosoma cruzi.
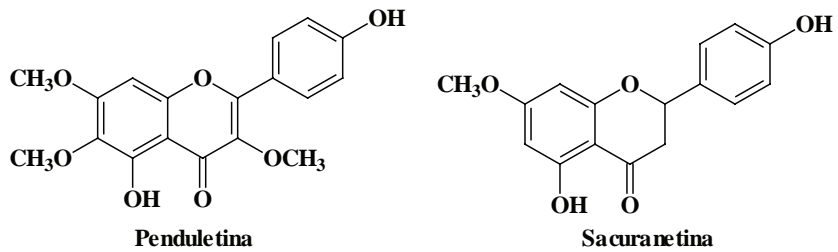<smiles>O=c1cc(-c2ccc(Cl)cc2)oc2c(Cl)c(O)c(Cl)c(Cl)c12</smiles><smiles>O=c1cc(-c2ccc(O)c(O)c2)oc2cc(O)cc(O)c12</smiles><smiles>N#CCOc1cc(O)cc2oc(-c3ccc(O)cc3)cc(=O)c12</smiles><smiles>[R16]Oc1c([R4])c(O)c2c(=O)c([R1])c(-c3ccc([R16])c([R6])c3)oc2c1[R4]</smiles>

1: $\mathbf{R}_{\mathbf{1}}=\mathbf{R}_{\mathbf{2}}=\mathbf{R}_{\mathbf{4}}=\mathrm{R}_{\mathbf{5}}=\mathbf{R}_{6}=\mathrm{H} ; \mathbf{R}_{\mathbf{3}}=\mathrm{Me}$

6: $R_{1}=R_{2}=R_{3}=R_{4}=R_{5}=R_{6}=H$

7: $\mathbf{R}_{\mathbf{1}}=\mathrm{OMe} ; \mathbf{R}_{2}=\mathbf{R}_{3}=\mathbf{R}_{4}=\mathbf{R}_{5}=\mathbf{R}_{6}=\mathbf{H}$

8: $\mathbf{R}_{1}=\mathrm{OMe} ; \mathrm{R}_{2}=\mathrm{R}_{3}=\mathrm{R}_{4}=\mathrm{H} ; \mathrm{R}_{5}=\mathrm{R}_{6}=\mathrm{OH}$

9: $\mathbf{R}_{1}=\mathbf{R}_{2}=\mathbf{R}_{3}=\mathbf{R}_{4}=H ; \mathbf{R}_{5}=\mathrm{OMe} ; \mathbf{R}_{6}=\mathrm{OH}$

10: $\mathbf{R}_{\mathbf{2}}=\mathbf{R}_{\mathbf{3}}=\mathrm{R}_{\mathbf{5}}=\mathrm{H} ; \mathbf{R}_{\mathbf{2}}=\mathrm{R}_{\mathbf{4}}=\mathrm{Gli} ; \mathrm{R}_{\mathbf{6}}=\mathrm{OH}$<smiles>[R20]c1cc(O)c2c(=O)c([R4])c(-c3ccccc3)oc2c1</smiles>

2: $\mathbf{R}_{1}=\mathbf{H} ; \mathbf{R}_{2}=\mathrm{Me}$

3: $\mathbf{R}_{1}=\mathrm{OH} ; \mathrm{R}_{\mathbf{2}}=\mathrm{H}$

4: $\mathbf{R}_{1}=O A c ; \mathbf{R}_{2}=H$

5: $\mathbf{R}_{\mathbf{1}}=\mathbf{R}_{\mathbf{2}}=\mathbf{H}$

Figura 4. Flavonóides com atividade anti-Trypanosoma cruzi.

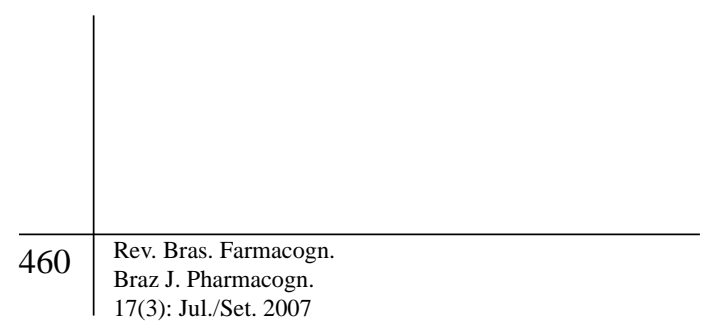




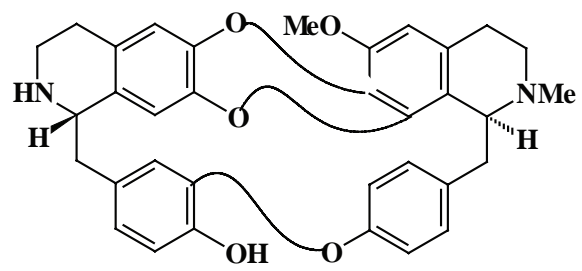

Cocsolina

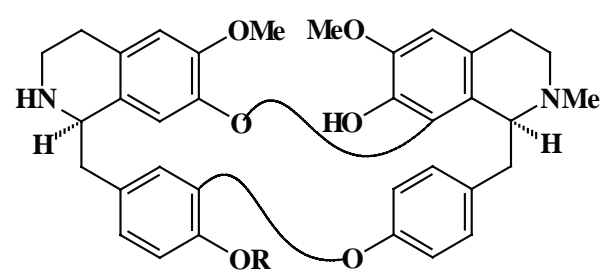

$\mathbf{R}=$ Me: Dafnandrina $\mathbf{R}=\mathbf{H}$ : Dafnolina<smiles>COc1ccc(COc2cc(C[C@H]3c4cc(OC)c(OC)cc4CCN3C)ccc2OC)cc1OC</smiles>

\section{Limacina}<smiles>COC1=C2C=C[C@@H](Cc3ccc(OC)cc3)NCCC(=C1)c1cc(OC)ccc1C[C@@H]1c3cc(c(O)cc3CCN1C)CCCO2</smiles>

Girocarpina<smiles>COc1ccc(COc2ccc(C[C@H]3c4cc(O)c(OC)cc4N(C)CC[C@H]3C)cc2)cc1</smiles>

Isochondrodendrina

Figura 5. Alcalóides com atividade anti-Trypanosoma cruzi.

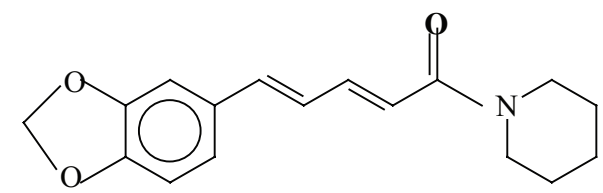

Piperina

Figura 6. Piperina de Piper nigrun com atividade anti-Trypanosoma cruzi.<smiles>CCCc1ccc2ccccc2n1</smiles>

2-n-Propilquinolina<smiles>C/C=C/c1ccc2ccccc2n1</smiles>

Chimanina B<smiles></smiles>

Chimanina D

Figura 7. Alcalóides Galipea longiflora com atividade anti-Trypanosoma cruzi. 


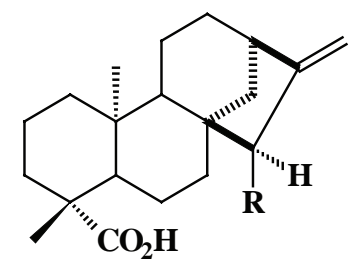

$R=$ H: Ácido caurenóico $\mathbf{R}=$ OAc: Ácido xilópico<smiles>CC1(C)CCC[C@]2(C)[C@H]3CC[C@@](C)(O)[C@@H]3CC[C@@H]12</smiles>

17-Hidroxicauranol<smiles></smiles>

Caurenol<smiles>CC12C[C@H]3C[C@]1(CCC1[C@](C)(C(=O)O)CCC[C@@]13C)C2</smiles>

Ácido traquilobânico

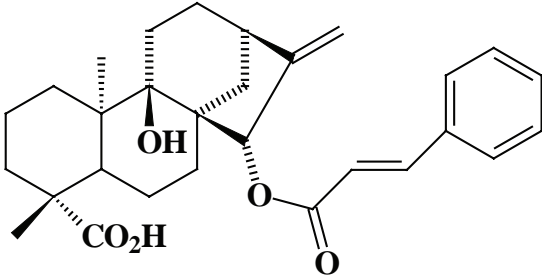

Ácido ent-9- $\alpha$-hidroxi-15- $\beta$-E-cinamoiloxi-16-cauren-19-óico<smiles>COC1=C(C(C)C)C=C2C(=O)O[C@@]34CCCC(C)(C)C(C3)C[C@]24C1=O</smiles>

Komarovspirona

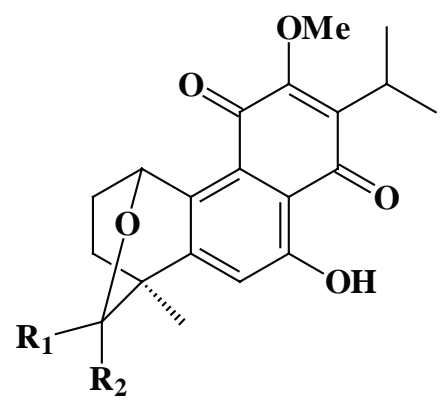

Dracocequinona A: $R_{1}=R_{2}=H$ Dracocequinona $B: R_{1}=R_{2}=0$<smiles>CC1=CCC2C(C)(C)CCC[C@]2(C)[C@H]1C[C@H](O)C1=CC(=O)O[C@H]1O</smiles>

ent-16-Hidroxilabda-7,13-dien-15,16-olídeo<smiles>CC1=C(O)C(=O)C=C2C1=CC=C1[C@@]3(C)CCC4(C)CC(=O)C(C)CC4[C@@]3(C)CC[C@@]21C</smiles>

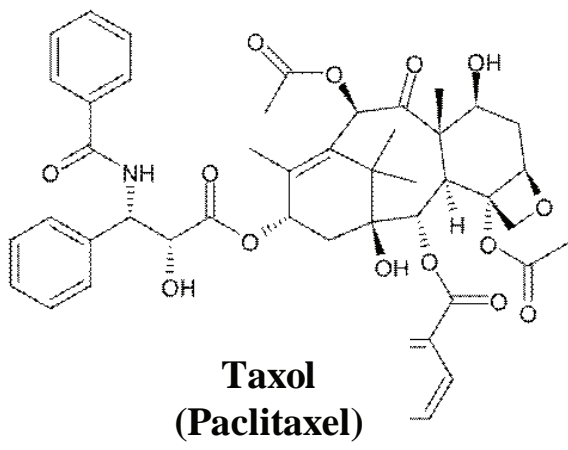




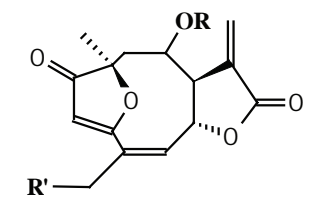

$\mathbf{R}^{\prime}=\mathbf{H}, \mathbf{R}=$ : $_{15}$

$\mathbf{R}^{\prime}=\mathrm{OH}, \mathrm{R}=\mathrm{H}_{\text {: Goiazensolídeo }}^{\mathrm{O}}$

$\mathbf{R}^{\prime}=\mathbf{H}, \mathbf{R}=$ : Licnofolídeo

$\mathbf{R}^{\prime}=\mathrm{OH}, \mathrm{R}=$ : Licnoforolídeo $\mathrm{A}$<smiles>C=C1C(=O)O[C@H]2C1C(O)CC(=C)[C@H]1C[C@@H](OC(C)=O)C(=C)[C@H]12</smiles>

8- Hidroxizaluzanina<smiles>C=C(CO)C(=O)O[C@H]1CC(=C)C2C[C@H](O)C(=C)C2[C@H]2OC(=O)C(=C)C12</smiles>

Cina ropicrina<smiles>C=C1CC/C=C(/C(=O)O)CCC2CC1(C)C2(C)C</smiles>

Ácido licnofóico

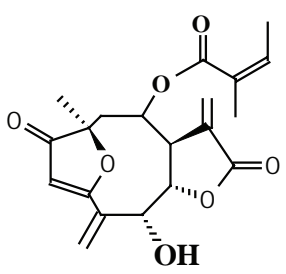

Isocentraterina

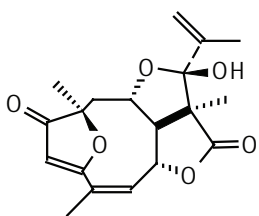

Eremantolídeo C
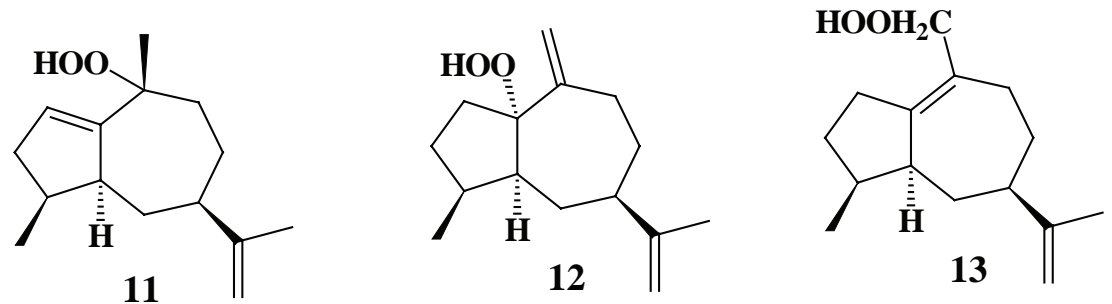

Figura 8. Terpenóides com atividade anti-Trypanosoma cruzi.<smiles>C=CC(C)(C)c1cc(O)c2oc3c4c(ccc3c(=O)c2c1O)C=CC(C)(C)O4</smiles><smiles>CC(C)=CCc1c(O)c(CC=C(C)C)c2oc3c(O)cccc3c(=O)c2c1O</smiles>

Figura 9. Xantonas com atividade anti-Trypanosoma cruzi. 
<smiles>COC1=C(OC)C(OC)CC(C2OC(c3cc(OC)c(OC)c(OC)c3)C(C)C2C)C1</smiles>

Grandisina<smiles>COC1=C(OC)C(OC)CC([C@H]2O[C@@H](c3cc(OC)c4c(c3)OCO4)C(C)C2C)C1</smiles>

14<smiles>COC1=CC(C2OC(c3cc(OC)c4c(c3)OCO4)C(C)C2C)CC2=C1OCO2</smiles>

15

Figura 10. Neolignanas com atividade anti-Trypanosoma cruzi.<smiles>CC(=O)C1=C(O)C=C2Oc3c(C(C)=O)c(O)c(C)c(O)c3C2(C)C1=O</smiles>

Figura 11. Benzofuranodiona com atividade anti-Trypanosoma cruzi.

161.

Dias JCP, Wendel S 1992. Transfusion transmitted Chagas' Disease. In S. Wendel, Z. Brener, M. E. Camargo and A. Rassi (ed), Chagas' disease (American Trypanosomiasis): Its impact on transfusion and clinical medicine. ISBT BraziL’92, São Paulo.

Dias JCP 2006. Notas sobre o Trypanosoma cruzi e suas características bio-ecológicas, como agente de enfermidades transmitidas por alimentos. Rev Soc Bras Med Trop 39: 370-375.

Fairlamb AH 1994. Trypanotione metabolism in Trypanosoma cruzi. Mem I Oswaldo Cruz 89 (Suppl. I): 37.

Feresin GE, Tapia A, Sortino M, Zacchino S, Arias AR, Inchausti A,Yaluff G, Rodriguez J, Theoduloz C, Schmeda-Hirschmann G 2003. Bioactive alkyl phenols and embelin from Oxalis erythrorhiza. $J$ Ethnopharmacol 88: 241-247.

Ferreira HO 1990. Tratamento da forma indeterminada da doença de Chagas com nifurtimox e benznidazol. Rev Med Trop 23: 209-211.

Fournet A, Muñoz V, Roblot F, Hocquemiller R, Cave A, Gantier JC 1993. Antiprotozoal activity of dehydrozaluzanin $\mathrm{C}$, a sesquiterpene lactone isolated from Munnozia maronii (Asteraceae). Phytother Res 7: 111-115.

Fournet A, Rojas de Arias A, Inchausti A, Ascurrat M, Fleitas N, Rodriguez E 1994. In vitro activity and mutagenicity of bisbenzilisoquinolines and quinones against Trypanosoma cruzi Trypomastigotes. Phytother Res 8: $141-144$

Grael CFF, Albuquerque S, Lopes JL 2005. Chemical constituents of Lychnophora pohlii and trypanocidal activity of crude plant extracts and of isolated compounds. Fitoterapia 76: 73-82.

Kiuchi F, Matsuo K, Ito M, Qui TK, Honda G 2004. New sesquiterpene hydroperoxides with trypanocidal activity from Pogostemon cablin. Chem Pharm Bull 52: 1495-1496.

Leite JR, Silva LP, Rodrigues MI, Prates MV, Brand G, Lacava BM, Azevedo RB, Bocca A, Albuquerque S, Bloch Jr C 2005. Phylloseptins: a novel class of anti-bacterial and anti-protozoan peptides from the Phyllomedusa genus. Peptides 26: 565-573.

Leite JPV, Lombardi JA, Chiari E, Oliveira AB 2001. Isolamento biomonitorado de uma substância tripanossomicida de Arrabidaea triplinervia (Bignoniaceae), o ácido ursólico. Rev Bras Farmacogn 11: 77-87.

Martins RCC, Lago JHG, Albuquerque S, Kato MJ 2003. Trypanocidal tetrahydrofuran lignans from inflorescences of Piper solmsianum. Phytochemistry 64: 667-670.

Morelo A, Lipchenca I, Cassels BK, Speisky H, Aldunate J, Repetto Y, 1994. Trypanocidal effect of boldine and related alkaloids upon several strains of Trypanosoma cruzi. Comp. Biochem Physiol Pharmacol Toxicol Endocrinol 107: 367-371.

Nascimento AM, Chaves JS, Albuquerque S, Oliveira DCR 2004. Trypanocidal properties of Mikania stipulacea and Mikania hoehnei isolated terpenoids. Fitoterapia 75: 381-384.

Oliveira AB, Saúde DA, Perry KSP, Duarte DS, Raslan DS, Boaventura MAD, Chiari E 1996. Trypanocidal Sesquiterpenes from Lychnophora Species. Phytother Res 10: 292-295.

Ribeiro A, Santos LMST, Romanha AJ, Veloso DP, Zani CL 
1997. Trypanocidal flavonoids from Trixis vauthieri J Nat Prod 60: 836-838.

Ribeiro TS, Lima LF, Previato JO, Previato LM, Heise N, Lima ME. 2004. Toxic effects of natural piperine and its derivatives on epimastigotes and amastigotes of Trypanosoma cruzi. Bioorg Med Chem Lett 14: 3555-3558.

Saúde DA, Raslan DS, de Souza Filho JD, de Oliveira AB 1998. Constituents from the aerial parts of Lychnophora trichocarpa. Fitoterapia 69: 90-91.

Schinor EC, Salvador MJ, Ito IY, Albuquerque S, Dias DA 2004. Trypanocidal and antimicrobial activities of Moquinia kingii. Phytomedicine 11: 224-229.

Schmunis GA 1999. Prevention of transfusional Trypanosoma cruzi infection in Latin America. Mem I Oswaldo Cruz 94 (Suppl I): 93-101.

Scio E, Ribeiro A, Alves TM.A, Romanha AJ, Filho JDS, Cordell GA, Zani CL 2003. Diterpenes from Alomia myriadenia (Asteraceae) with cytotoxic and trypanocidal activity. Phytochemistry 64: 11251131.

Sigman L, Sanchez VM, Turjanski AG 2006. Characterization of the farnesyl pyrophosphate synthase of Trypanosoma cruzi by homology modeling and molecular dynamics. J Mol Graphics Modell 25: 345-352.

Takahashi JA, Boaventura MAD, Oliveira AB, Chiari E, Vieira HS 1994. Isolamento e atividade tripanossomicida de diterpenos caurânicos de Xylopia frutescens Aubl. $17^{a}$ Reunião da Sociedade Brasileira de Química, Caxambu.

Takahashi JA, Vieira HS, Silva EA, Boaventura MAD, Oliveira AB, Egler Chiari E 2002. Preparation and activity of diterpenoids against trypomastigotes of Trypanosoma cruzi. Rev Bras Farmacogn 12(Supl.): 118-120.

Takeara R, Albuquerque S, Lopes NP, Lopes JLC 2003. Trypanocidal activity of Lychnophora staavioides Mart.(Vernonieae, Asteraceae). Phytomedicine 10: 490-493.

Tavares KG 1990. Estudo químico de Lychnophora vilosissima (Compositae), Piper aduncum e Piper superba (Piperaceae). Belo Horizonte: Universidade Federal de Minas Gerais, 163p.

Uchiyama N, Ito M, Kiuchi F, Honda G, Takeda Y, Khodzhimatov OK, Ashurmetov OA 2004. A trypanocidal diterpene with novel skeleton from Dracocephalum komarovi. Tetrahedron Lett 45: 531533.

WHO 2002. Tech. Rep., Control of Chagas' disease 2002. 905:i. 